\title{
Exemples d'applications pédagogiques autour du kart électrique e-kart
}

\author{
T. LEQUEU (a), B. BIDOGGIA (b), A. SCHELLMANNS (c), Y. DERRIEN (a), N. GODEFROY (a) \\ E-mail : thierry.lequeu@univ-tours.fr \\ (a) IUT de Tours, département GEII, avenue Monge, Parc de Grandmont, 37200 TOURS \\ (b) L.M.P., Polytech Tours, 7 avenue Marcel Dassault, 37200 TOURS \\ (c) Polytech Tours, département Productique, 7 avenue Marcel Dassault, 37200 TOURS
}

\begin{abstract}
RÉSUMÉ : Les auteurs présentent ici deux exemples d'applications pédagogiques autour du e-kart (le kart électrique) du Département Génie Electrique et Informatique Industrielle de l'Institut Universitaire de Technologie de Tours. Initié depuis 2003, ce projet prend de l'ampleur avec la réalisation pour en 2007 d'un kart de compétition avec motorisation asynchrone basse tension. Le premier projet présenté ici concerne l'acquisition d'informations physiques sur le kart, de la transmission au sol de ces informations et de l'affichage par un ordinateur fixe. Le second développement traite de la réalisation de la commande vectorielle du moteur asynchrone alimenté par des batteries en $48 \mathrm{~V}$.
\end{abstract}

Mots clés : kart électrique, acquisition et transmission de données, commande vectorielle, moteur asynchrone

\section{1}

\section{INTRODUCTION}

Lors de l'édition 2005 du CETSIS, le département GEII de l'IUT de Tours a présenté le kart électrique comme support pédagogique pluridisciplinaire [1]. Depuis 2003, les évolutions successives du projet ont conduit à l'organisation du premier challenge «e-kart» à Joué-lès-Tours en 2006, avec la présentation d'un kart en $24 \mathrm{~V}$ possédant un moteur à courant continu de $6 \mathrm{~kW}$ [2]. Chez Speed $O$ Max à Clermont, lors du « eKart 2007 », les étudiants du «e-Kart GEII Tours Club » ont assemblé un e-kart de compétition avec un moteur asynchrone de $10 \mathrm{~kW}[3]$.

Fort des expériences de ces quelques années, l'IUT GEII de Tours a orienté ses développements dans les trois axes suivants :

1) la métrologie embarquée. Afin de dépasser le stade de l'empirisme dans le réglage du kart et pour avoir une vraie application pédagogique, il est indispensable de pouvoir mesurer quelques paramètres électriques et mécaniques sur le kart, avec comme objectif l'estimation de la puissance, de l'autonomie et de l'accélération du véhicule ;

2) la maîtrise du variateur pour moteur asynchrone. L'utilisation du hacheur réversible en courant étant bien maîtrisée pour l'utilisation de moteurs à courant continu sur le e-kart, il devient nécessaire d'étudier, de développer et de maîtriser la commande vectorielle du moteur asynchrone (et ultérieurement du moteur synchrone);

3) le kart pour handicapé. Cette voie de développement concerne différents projets autour du kart électrique à destination des personnes handicapées. L'étude porte sur la commande au volant de l'accélération et du freinage, l'assistance au pilotage à distance, et la recherche de solutions pour le pilotage par des personnes aveugles...

L'article présenté ici traite de façon détaillée de l'acquisition des données et de la transmission au sol (télémétrie) par le biais d'une liaison WiFi. Ce travail est réalisé par Yann DERRIEN, étudiant en Licence Professionnelle EICET de l'IUT de Valenciennes [4].

Nicolas GODEFROY étudie la commande vectorielle dans le cadre de son stage de Master 1 IUP STS/EEATS/GEII de l'Université Joseph Fourier de Grenoble [5].

L'article présente également les perspectives de développement d'un kart électrique bi-place bi-moteur pour handicapé.

\section{ACQUISITION ET TRANSMISSION DE DONNEES}

En ce qui concerne la transmission entre le véhicule (e-kart) et une station de travail fixe (type PC portable) par le biais de la liaison WiFi (802.11 a, b, g ou autre), le sujet se décompose en trois parties distinctes (Fig. 1). Il y a différents moyens de récupérer les informations qui nous intéressent, mais également différents moyens de les «convertir» en WiFi et de les transmettre (deuxième partie). La réception et le traitement des informations reçues constituent la troisième et dernière partie.

\subsection{Acquisition des grandeurs physiques}

Cette partie aborde les différents moyens de collecte des informations. Par exemple, ici le microcontrôleur utilisé est un ATmega8535 de chez Atmel, composant relativement flexible et programmable à partir d'un logiciel assez intuitif [6]. Il dispose de plusieurs interfaces tel un port SPI, l'UART, des CAN (Convertisseur Analogique Numérique) ainsi que plusieurs compteurs et entrées d'interruptions. Par le biais des compteurs et interruptions, on peut effectuer un compte tours grâce au capteur intégré au moteur du kart. Les différents CAN vont permettre de surveiller les tensions batteries, les courants (batteries et moteurs) à des intervalles réguliers pour effectuer les statistiques désirées. 

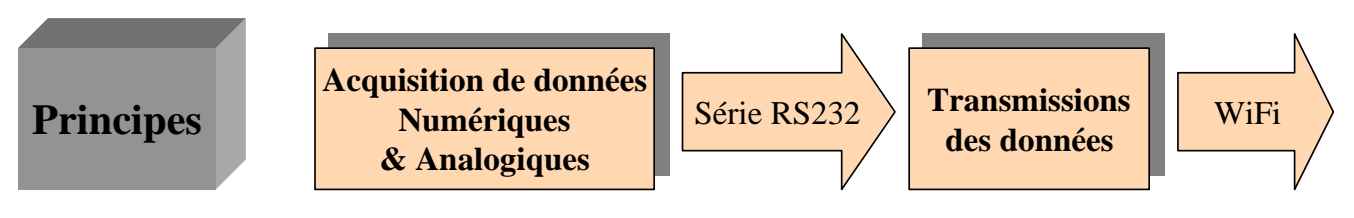

Traitements et affichages des données
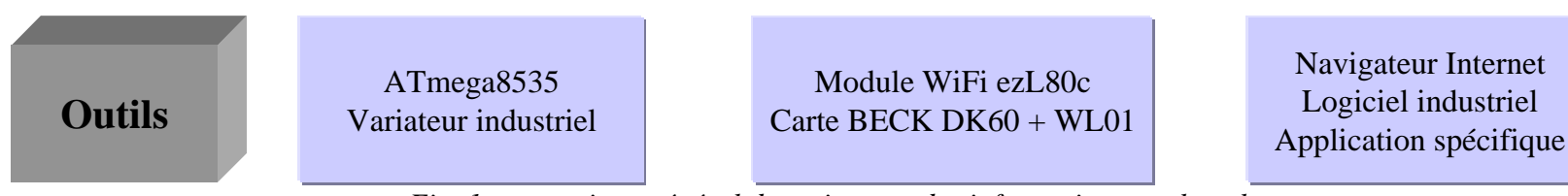

Fig. 1 : synoptique général du traitement des informations sur le e- kart.

L'avantage de l'utilisation du microcontrôleur est que ce principe est ouvert et peut permettre de créer des fonctions annexes avec un capteur adéquat et un traitement analogique ou numérique pour adapter le signal de sortie du capteur en une variable numérique stockée dans la mémoire du composant.

Une autre solution est de lire les informations issues des trames en sortie du variateur (modèle Curtis 1236-5301 dans notre cas [7]), trames qui transitent via une liaison série RS232. L'avantage de cette méthode est qu'elle permet de récupérer plus d'information sans avoir à mettre en œuvre un autre circuit électronique. De plus, on peut récupérer des informations telles que le couple développé par le moteur, et les paramètres internes au variateur comme le couple de recul maximum, la vitesse de rotation maximale du moteur, la position de la pédale, les températures... L'inconvénient majeur de ce système est que pour traiter les trames des variateurs (après les avoir émises en WiFi) il faut les décrypter, ce qui s'avère très difficile quand on ne dispose pas de la documentation adéquate.

\subsection{Transmission des données}

Cette partie aborde les deux principaux moyens de transmission WiFi. A noter que le protocole série RS232 standard a été choisi pour le transit des informations entre les moyens d'acquisition et de transmission. Des solutions avec un bus SPI, I2C, CAN, CSMA-CD auraient pu être envisagées.

Le premier composant utilisé est le module EzL80C. Il transmet directement les trames RS232 en WiFi, (mode Ad-Hoc ou infrastructure) [8]. Il est facilement paramétrable, dans un premier cas grâce à un cordon série et au logiciel fourni par Sollae, constructeur du module, puis directement par WiFi une fois le contact établi.

Le second composant est beaucoup plus élaboré que le premier module puisqu'il s'agit de la carte DK60 (Development Kit) de chez Beck@IPC avec le module WL01, le module WiFi adapté à la carte [9] (Fig. 2). Le module DK61 (DK60 + WL01) est plus puissant dans le sens où il peut gérer différents protocoles parmi lesquels un bus d'adressage direct, un bus I2C, deux ports Ethernet, deux ports RS232, deux BUS CAN (très utilisé dans l'électronique embarquée). Le tout est géré par un SC143, qui embarque un OS (Operating System) temps réel.

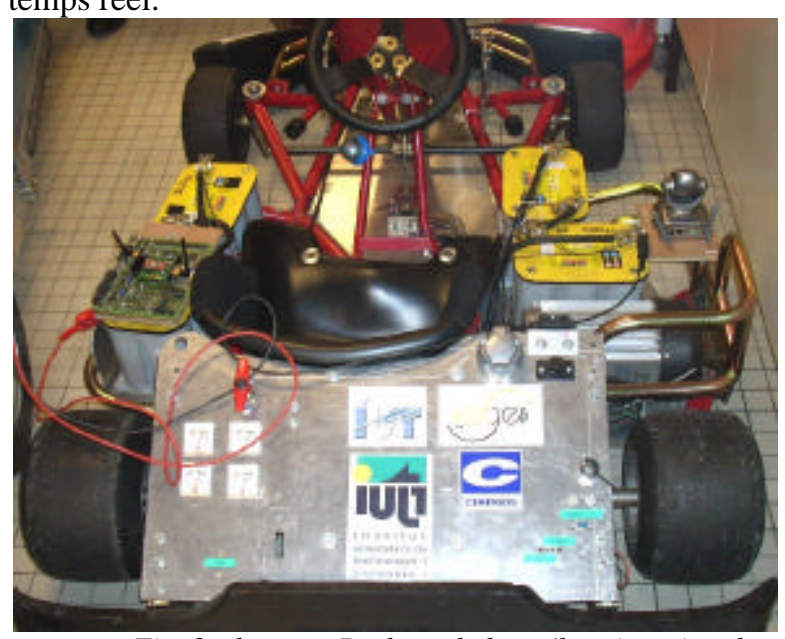

Fig. 2 : la carte Beck sur le kart électrique (sur la batterie de gauche).

Un avantage supplémentaire est que cette carte dispose de lecteur de carte SD/MMC et CF (Compact Flash), ce qui permet de stocker un nombre important d'informations en plus de les envoyer par WiFi.

Le SC143 qui embarque le RTOS (Système d'Exploitation Temps Réel) permet, de plus, un faible espace de stockage supplémentaire pour les programmes (ou tâches du RTOS), et permet aussi de stocker différents fichiers web (au format HTML). Cela facilite l'affichage direct des informations, sans avoir à installer un programme, ce qui facilite le traitement et l'installation d'applications sur PC (puisqu'il n'y a rien à installer excepté une clé USB Wifi et de se connecter sur une adresse IP type 192.168.0.2, celle du module WiFi WL01).

\subsection{Traitement des informations}

Pour le traitement des informations reçues, cela dépend bien évidemment du module d'émission choisi.

Pour l'EzL-80C, un logiciel fourni par le constructeur permet après avoir établi la liaison WiFi entre le module et le PC de créer un port COM virtuel afin de le traiter comme un port COM standard dans un logiciel de son choix. Il est possible alors d'utiliser le logiciel du variateur ou alors un logiciel développé en C, C++, Java, Pascal, Delphi ou autre. 
La carte DK61 de Beck peut également héberger une interface de type HTML (serveur web). Il suffit alors de créer la connexion WiFi, puis d'entrer l'adresse IP du module WL01. L'accès peut être sécurisé par un mot de passe supplémentaire. Mais des moyens plus complexes peuvent être mis en œuvre.

\section{COMMANDE VECTORIELLE POUR MOTEUR ASYNCHRONE}

La commande vectorielle est réalisée sur un moteur asynchrone qui équipe un e-kart. Le moteur asynchrone n'a besoin d'aucun entretien à l'inverse du moteur à courant continu où les balais doivent être changés assez fréquemment. Il est aussi plus facile d'obtenir la marche arrière.

\subsection{Principes de commande}

Plusieurs principes de fonctionnement existent sur les variateurs pour moteurs asynchrones et dépendent de la loi de modulation (Pulse Width Modulation PWM) appliquée sur l'onduleur. Il existe des lois en U/f simple et en U/f compensée. Cette dernière évite les problèmes de chutes de tension non négligeables dues à la résistance statorique pour les faibles vitesses.

La plus performante, mais néanmoins plus compliquée, est la commande vectorielle. Cette loi de commande a pour principe d'aligner l'axe (d) du référentiel de Park (champ tournant) avec le vecteur du flux rotorique. Avec cet alignement, les équations du moteur asynchrone deviennent les suivantes, dans le référentiel de Park :

$$
\begin{aligned}
V s d & =R s . I s d+\sigma . L s . \frac{d I s d}{d t}-w s . \sigma . L s . I s q+\frac{L \mu}{L r} \cdot \frac{d \Phi r d}{d t} \\
V s q & =R s . I s q+\sigma . L s . \frac{d I s q}{d t}+w s . \sigma . L s . I s d+w s . \frac{L \mu}{L r} \cdot \frac{d \Phi r d}{d t} \\
0 & =-L \mu . I s d+\Phi r d+\tau r . \frac{d \Phi r d}{d t} \\
0 & =-L \mu . I s q+w r . \tau r . \Phi r d
\end{aligned}
$$

En régime permanent, on obtient :

$$
\begin{aligned}
V s d & =R s . I s d-w s . \sigma . L s . I s q \\
V s q & =R s . I s q+w s . \sigma . L s . I s d \\
0 & =-L \mu . I s d+\Phi r d \\
0 & =-L \mu . I s q+w r . \tau r . \Phi r d
\end{aligned}
$$

Les expressions du flux, de la vitesse de rotation et du couple moteur sont alors données par :

$$
\begin{aligned}
& \Phi r d=L \mu . I s d \\
& w r=\frac{L \mu \cdot I s q}{\tau r . \Phi r d} \\
& C m=3 . p \cdot \frac{L \mu}{L r} . \Phi r d . i s q
\end{aligned}
$$

La valeur de Isd règle la valeur du flux rotorique $(\mathrm{r}=\mathrm{rd})$. Isd est le courant dit magnétisant. A rd constant (à flux donné), Isq règle la valeur du couple. Isq est le courant dit de charge. La commande vectorielle permet donc de reconstituer par la commande une MCC, c'est-à-dire un découplage de l'état magnétique et de l'état de charge. Un asservissement sur les courants Isd et Isq permet de contrôler le flux rotorique et le couple moteur.

Pour notre application, nous voulons que le moteur fournisse un couple dépendant de la position de la pédale. Notre référence Isq (couple) sera donc donnée par la position de la pédale. La référence de Isd sera fixée pour obtenir dans le moteur un flux nominal. Celui-ci devra être établi avant la demande d'un couple au moteur pour diminuer les courants de démarrage. Pour obtenir le courant statorique désiré (Isd et Isq), l'onduleur va créer une tension statorique (Vsd, Vsq) dépendante des équations précédentes. Il est nécessaire d'avoir la position du rotor pour passer du référentiel de Park à un référentiel fixe (stator). Pour l'obtenir, il nous faut mesurer la vitesse du rotor et également mesurer les courants réels Isd, Isq.

\subsection{Un peu de technique}

\subsubsection{Capteurs de courant}

Nous plaçons deux capteurs de courant LEM HAS 200 qui supportent +/- 600 A sur les câbles d'alimentation du moteur (Fig. 5). Ces deux courants sont suffisants pour reconstituer le courant statorique dans le référentiel de Park (système triphasé équilibré).

\subsubsection{Capteur de vitesse et de position}

Pour mesurer la vitesse, le moteur utilisé possède un codeur QEP 32 pas intégré dans un roulement à bille. Les impulsions nous permettent de retrouver la vitesse du rotor.

\subsubsection{Le moteur asynchrone}

Le moteur installé sur le kart est un moteur asynchrone distribué par la société Speed O Max [11] (Fig. 3). Celui-ci possède une tension nominale de $28 \mathrm{~V}$. Il est en basse tension pour plus de sécurité et a été conçu pour faciliter l'alimentation par batterie en $48 \mathrm{~V}$. Le moteur peut être «poussé » à une puissance de $10 \mathrm{~kW}$, soit des courants de phase d'environ $240 \mathrm{~A}$.

Les composants de l'onduleur de tension formant la partie puissance du variateur doivent donc supporter de fortes intensités.

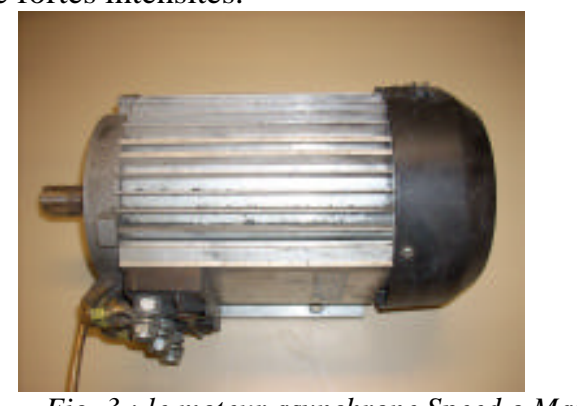

\subsubsection{L'onduleur triphasé}

L'onduleur est formé par trois modules MOSFET APTM20AM06SG, bras de pont supportant un courant de 372 A et une tension de $200 \mathrm{~V}$ pour une résistance $\mathrm{R}_{\mathrm{DSON}}$ de $6 \mathrm{mOhms} \mathrm{[12]} \mathrm{(Fig.} \mathrm{4).}$ 


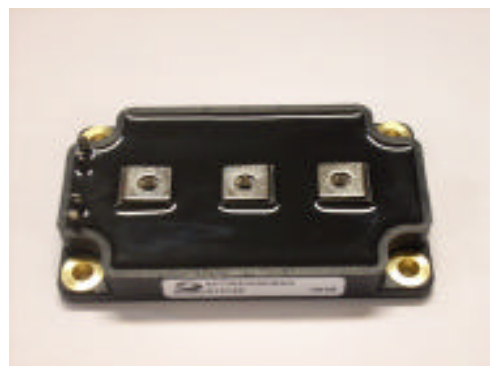

Fig. 4 : le bras de MOSFET en module APT.

Les modules sont assemblés mécaniquement sur un dissipateur commun (Fig. 5). Un «bus bar» en cuivre réalise les liaisons entre les modules et les condensateurs de découplage. Les câbles de sortie vers le moteur (section de $50 \mathrm{~mm}^{2}$ ) sont directement vissés sur les bornes des modules.

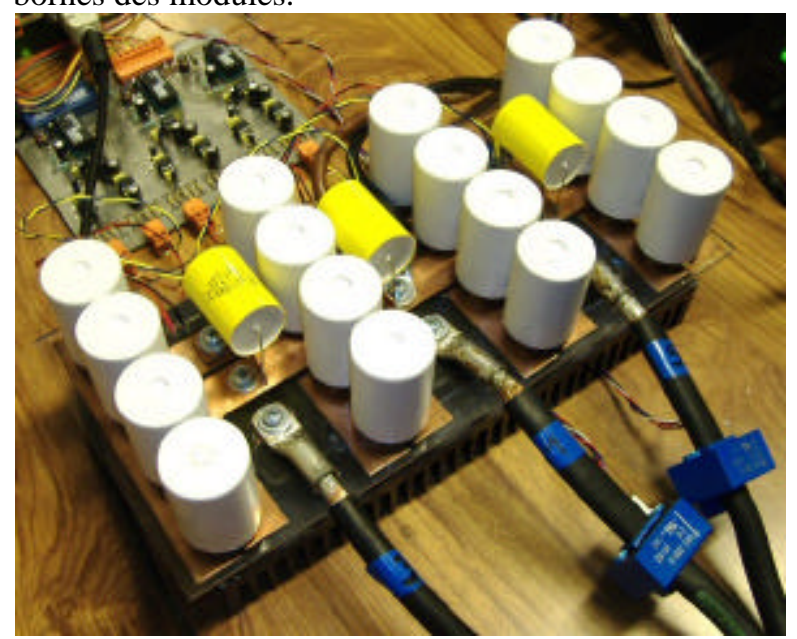

Fig. 5 : assemblage de l'onduleur triphasé.

Les transistors de puissance sont commandés par une carte «driver» (Fig. 6). Cette carte est alimentée directement à partir des batteries en $48 \mathrm{~V}$. Les commandes des transistors «flottant» sont alimentées individuellement afin de pouvoir supporter un rapport cyclique unitaire sur une période du courant moteur.

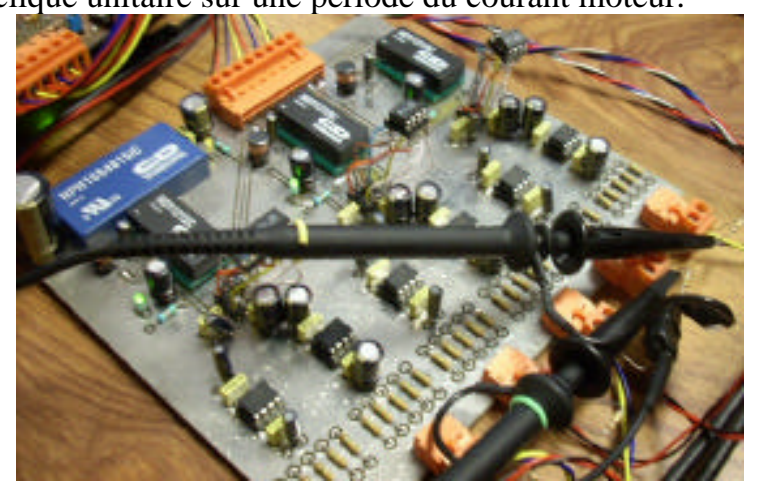

Fig. 6 : carte drivers des modules MOSFETS.

\subsubsection{La carte numérique de contrôle}

Le processeur utilisé pour faire les calculs, les changements de référentiel et la commande des bras de MOSFET est un DSP de chez TEXAS INSTRUMENT, le C2812, de la famille de C2xxx [13]. Cette famille est dédiée aux applications motorisées. Il pos- sède de nombreuses entrées $\mathrm{A} / \mathrm{N}$ (x16) afin de récupérer les différents courants et tensions, et des générateurs PWM. Il dispose d'entrées dédiées pour les codeurs de vitesse CAP/QEP.

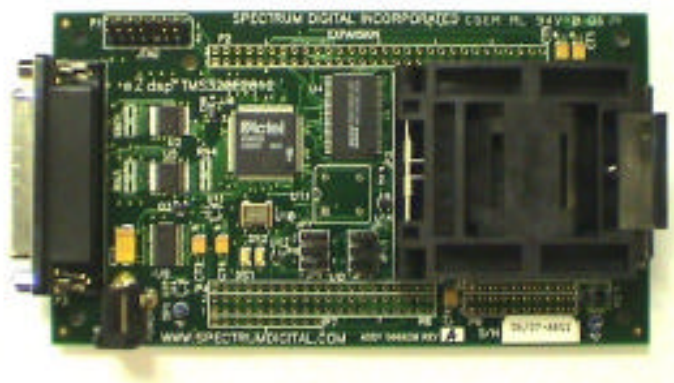

Fig. 7 : le Starter Kit eZdsp TMS320 F2812.

\subsection{Un peu de logiciel}

Pour simplifier la réalisation des applications, Texas Instrument a mis à disposition des blocs de fonctions préprogrammées. Ils permettent de réaliser simplement tous les changements de référentiel, la mise en place des correcteurs ou encore la création des lois PWM. Le synoptique «Fig. 9 » présente le schéma bloc de la commande vectorielle avec les différentes fonction de la librairie Texas Instrument.

\subsection{Essai de l'onduleur}

Afin de visualiser le bon fonctionnement des générateurs PWM, un filtre R-C est utilisé pour extraire la valeur moyenne du signal (courbes Ch1 et Ch1 Fig. 8). En boucle fermée, la régulation des courants de phase impose des courants sinusoïdaux (exemple de la courbe Ch3 Fig. 8). L'essai présenté ici à été réalisé sous tension réduite, limitée par l'alimentation de puissance $30 \mathrm{~V}$, délivrant 40A max.

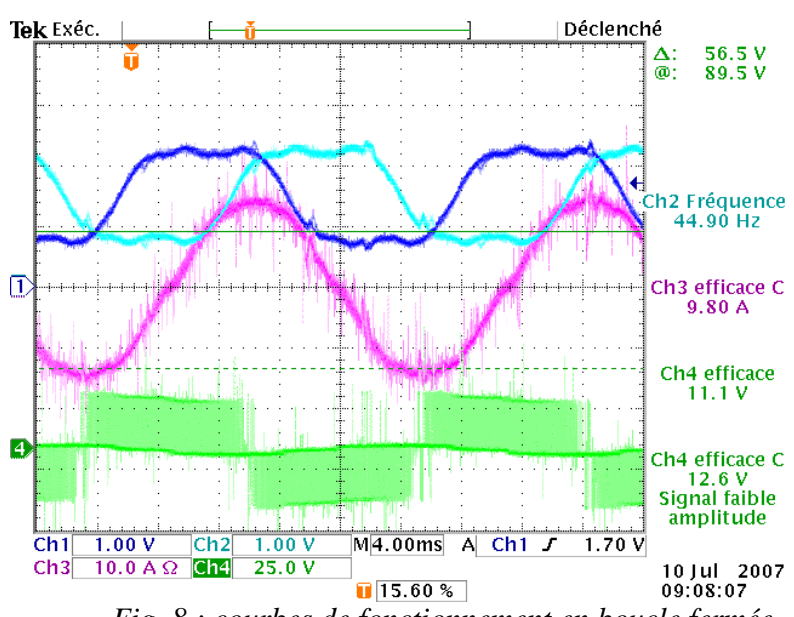

Fig. 8 : courbes de fonctionnement en boucle fermée. 


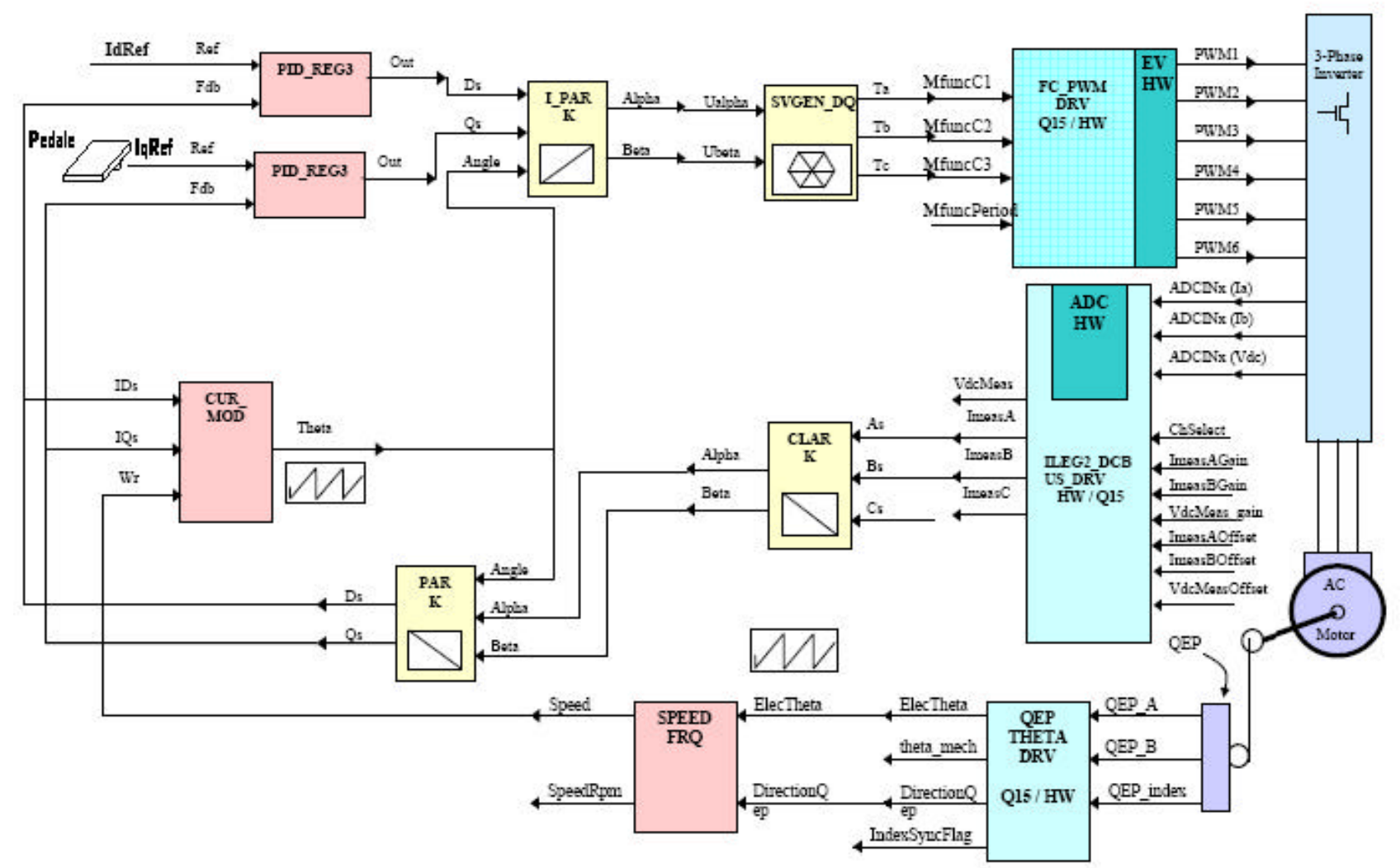

Fig. 9 : synoptique général de la commande vectorielle.

\section{4}

\section{PERSPECTIVES}

Le kart électrique est un outil d'intégration des personnes handicapées dans la pratique du sport mécanique. Outre les demandes multiples de réalisation de karts électriques monoplaces avec des commandes adaptées pour les handicapés, le bi-place s'inscrit dans l'application de conduite accompagnée (Fig. 10), le passager n'ayant pas la possibilité de conduire.

Il sera également utilisé pour le développement du kart pour aveugle, avec capteurs de distance et transmission sonore de l'information. Il est très intéres- sant également pour la pratique du kart à deux, avec les enfants ou en couple.

Ce kart bi-moteur est un petit retour vers la motorisation à courant continu. En effet, la principale difficulté réside dans la gestion correcte du différentiel électrique [14], d'où le choix d'une motorisation plus simple. La commande des moteurs se faisant par le biais de hacheurs réversibles en courant (Fig. 11), le pilotage du différentiel peut se faire par la régulation du courant dans les moteurs, mais aussi par la régulation de la vitesse de rotation.
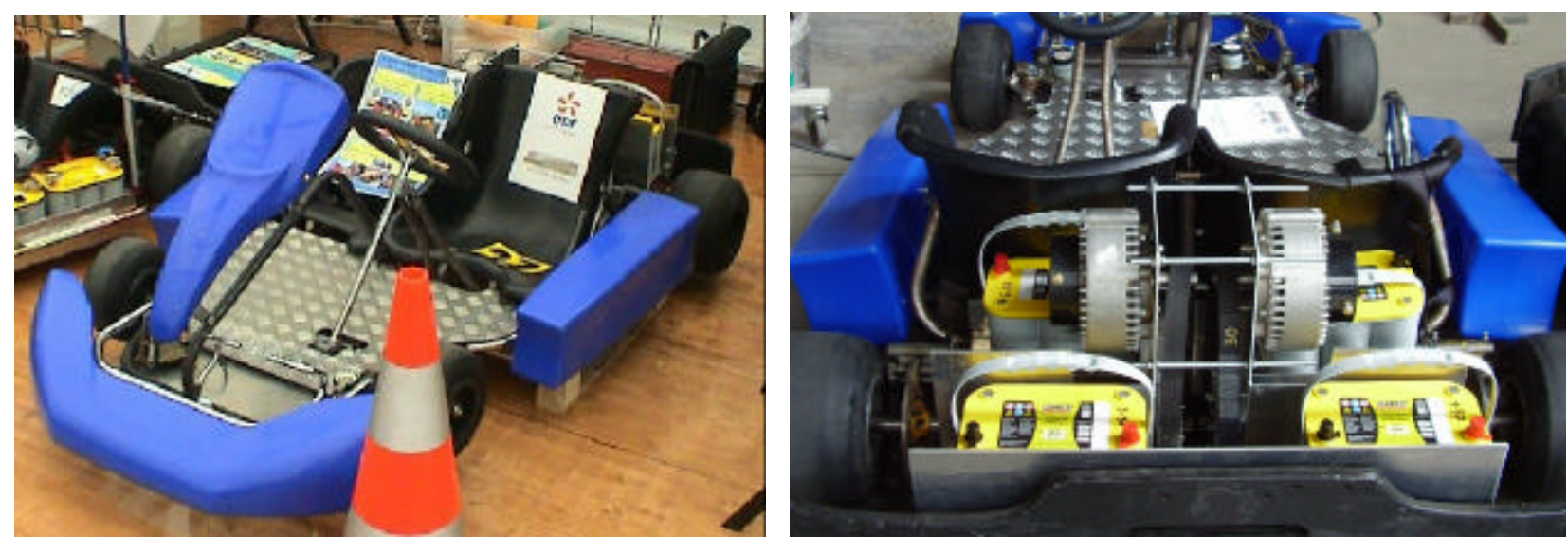

Fig. 10 : le kart électrique bi-place pour handicapé, bi-moteur pour un différentiel électrique. 
La structure de puissance utilisée ici sera identique à celle développée par les étudiants en 2005 et 2006 (Fig. 11 et Fig. 12). Le double hacheur de puissance sera assemblé sur un dissipateur commun.

La loi de comportement du différentiel électrique sera programmée sur un microcontrôleur. La transmission aux sols des informations sera ici un moyen très utile de vérifier le bon comportement du projet final.

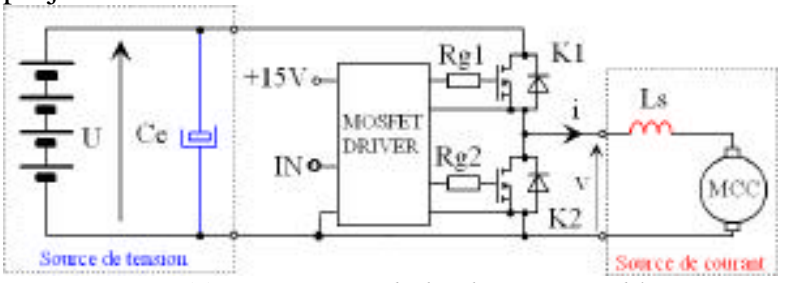

Fig. 11 : synoptique du hacheur réversible en courant pour moteur à courant continu.

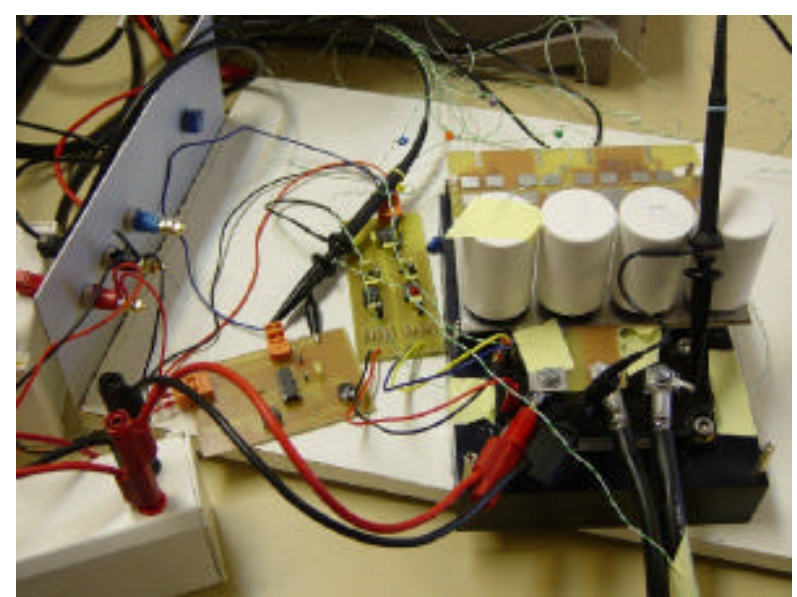

Fig. 12 : hacheur DC-DC avec un module MOSFET APT $100 \mathrm{~V} 495 \mathrm{~A} 2,25 \mathrm{~m} \Omega$.

\section{CONCLUSION}

Depuis 2003, le département GEII de l'IUT de Tours utilise le kart électrique comme support pédagogique pluridisciplinaire. Le succès des challenges pédagogiques de 2006 et de 2007 montrent très nettement l'intérêt du produit et la nécessité de développer les aspects de la compétition.

Pour une exploitation optimale du «e-kart», la télémétrie est indispensable pour bien comprendre les tenants et les aboutissants de la motorisation électrique d'un kart.

L'avantage de la transmission WiFi n'est plus à prouver dans le domaine des équipements mobiles. Les solutions à base de microcontrôleurs permettent à chacun d'ajouter des fonctions, autorisant une évolution ou une utilisation au bon grès de chacun et adaptable sur d'autres véhicules et avec d'autres capteurs.

L'arrivée des moteurs asynchrones dans les applications basse tension (ici $48 \mathrm{~V}$ ) nécessite l'adaptation des lois de commande vectorielle, afin de tenir compte des spécificités faible tension fort courant.

Ce support pédagogique est une mine de ressources, aussi bien techniques que sociales, véhiculant une image positive et moderne de l'Electrotechnique, de l'Electronique et de l'Automatique, intégrsé dans une démarche de développement durable.

\section{REMERCIEMENTS}

Les auteurs tiennent à remercier Stanislas RENARD, de l'IUT GMP du Mans, qui a réalisé l'usinage du dissipateur et la découpe du «bus bar » en cuivre et également Christophe BOUCHERIE, de l'IUT GMP du Mans, pour la réalisation mécanique du kart bi-place, sans oublier Denis COURATIN du Service Technique de la Faculté des Sciences de l'Université François Rabelais de Tours.

\section{BIBLIOGRAPHIE}

[1] T. LEQUEU, Un support pédagogique pluritechnologique : le kart électrique, CETSIS-EEA 2005, 5ième Colloque sur l'Enseignement des Technologies et des Sciences de l'Information et des Systèmes, Club EEA, 25-27 octobre 2005.

[2] http://www.e-kart.fr/2006/ - Le site du Chalenge pédagogique e-Kart 2006.

[3] http://www.e-kart.fr/2007/ - Le site du Chalenge pédagogique e-Kart 2007.

[4] Y. DERRIEN, Acquisitions de données et transmission par liaison WiFi pour karts électriques, stage de Licence Professionnelle E.I.C.E.T., IUT de Valenciennes, du 26 février au 15 juin 2007.

[5] N. GODEFROY, Développement de la commande vectorielle d'un moteur asynchrone basse tension $48 \mathrm{~V} 10 \mathrm{~kW}$, stage de Master 1 IUP STS/EEATS/GEII, Université de Grenoble 1, du 2 avril au 27 juillet 2007 .

[6] http://www.atmel.com/ voir le microcontrôleur ATmega8535.

[7] http://www.curtisinst.com/ - Variateurs industriels pour moteur asynchrone.

[8] http://www.lextronic.fr/sollae/ezl80c.htm - Module RS232 / WiFi.

[9] http://www.beck.com - Carte de développement temps réel DK60.

[10] http://www.lem.fr - Capteurs de courants isolés à effet Hall.

[11] http://www.speedomax.fr - Fabricant et exploitant de kart électrique.

[12] http://www.microsemi.com/ - Advanced Power, composants semi-conducteurs de puissance.

[13] http://www.ti.com/ voir TMS320C2812.

[14] M. CHAVES, L. PERRO, A. ROQUE, D. PRATA, J. MAIA, P. VERDELHO, J. ESTEVES, Control of an Electrical Kart With Two Independent Motors, EPE Proceedings, Septembre 1999. 